\title{
The Formation and Evolution of a Ring-filament Associated with Newly-emerging Flux
}

\author{
Suli Ma, Yunchun Jiang and Huadong Chen \\ National Astronomical Observatories/Yunnan Observatory, Chinese Academy of Sciences, \\ Kunming 650011, China \\ email: msli@ynao.ac.cn
}

\begin{abstract}
Using the data from BBSO, YNAO and SOHO/MDI we studied the formation and evolution of a ring-filament which was located in NOAA AR09470 from May 21 to 24, 2001. We found that: (1) the ring-filament's formation was well associated with newly-emerging flux. (2) all the eruptions of the filament happened during the decay of the emerging bipoles.
\end{abstract}

Keywords. Sun: ring-filament, magnetic flux, activity

\section{Observations}

In this paper we studied the formation and eruptions of a ring-filament which was well associated with newly-emerging flux. Figure 1 showed the ring-filament's formation and its first two times of eruptions. We also gave some MDI magnetograms and calculated the flux in the region marked with the white box in Figure 2.

On May 20, a magnetic bipole emerged in weak positive magnetic field and more bipoles appeared in the same region subsequently, which formed the NOAA Active Region 09470 later. The filament formed later than the emerging of magnetic flux. From the Figure 1 we can see that no filament segment formed at 14:27 UT on May 21. By 15:45 UT some filament segments had formed obviously. Comparing the $\mathrm{H} \alpha$ images and MDI images we can find that: some filament segments between the negative flux of the emerging bipoles and the surrounding positive flux formed earlier; while other filament segments formed later in the neutral line between the two poles. The filament segments changed their figures as the magnetic fields changed. At about 00:00 UT on May 22, when the emering flux reached its maximum, those filament segments closed and then a ring-filament formed eventually. During the formation of the filament segments, very faint cancellation activity happened at the left frontier of the bipoes.

From the profile of flux showed in the right panels of Figure 2, we can see that: (1) the flux reached its maximum at 00:00 UT on May 22 and began to decrease after that time; (2) the ring-filament erupted several times and all eruptions happened during the descending period of flux. We showed the first two eruptions in Figure 1. One happened at about 14:22 UT on May 22 and the other at about 22:50 UT on May 22. The two eruptions both happened from the filament segments located between the different newly-emerging bipoles. The rest of eruptions were in the same way. After each eruption except for the last one, the ring-filament reformed again, although they might be a little different on figure. After the last eruption happened at about 23:00 UT on May 24, the ring-filament disappeared in $\mathrm{H} \alpha$ finally. Correspondingly, from Figure 2 we can see that: first, the two poles of bipoles separated gradually; second, magnetic cancellations took place not only among the newly-emerging bipoles but also between the newly-emerging flux and 


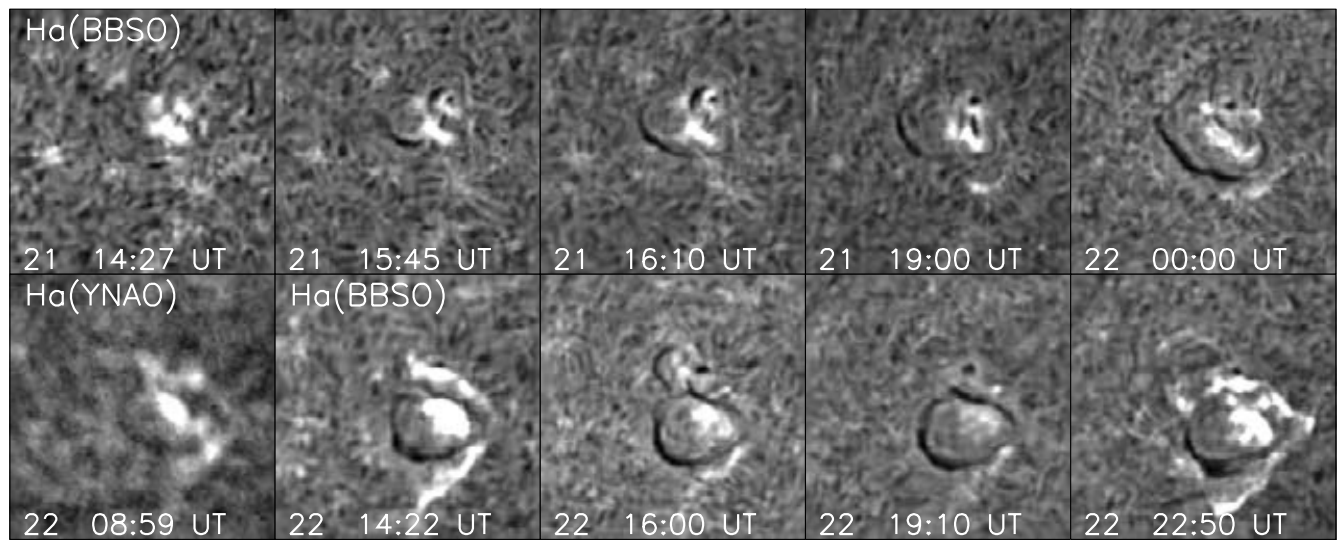

Figure 1. BBSO and YNAO $\mathrm{H} \alpha$ images showing the formation and evolution of the ring-filament. The field of view is 170"X170".
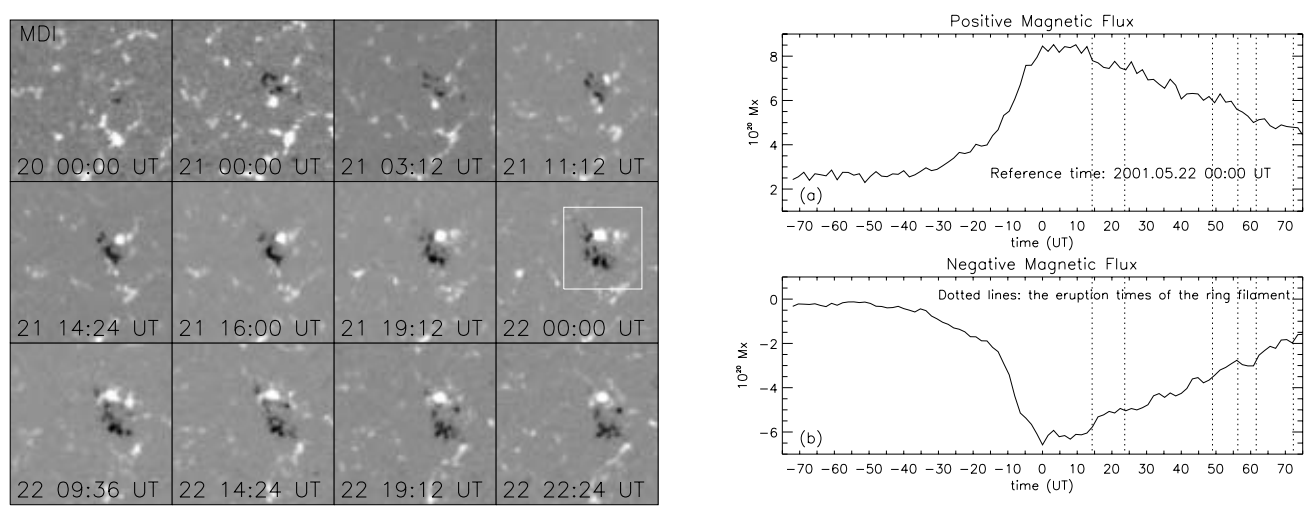

Figure 2. Left: MDI showing the evolution of magnetic field. The white box indicates the region where we calculate the flux. Right: Magnetic flux as a function of time. Reference time is 00:00 UT on May 22,2001. The dotted line indicates the time of eruptions of the ring-filament.

surrounding flux; last, the shearing movements happened between the couple poles of bipoles.

\section{Conclusions}

The formation of the ring-filament was well associated with newly-emerging flux. It seemed that the filament formed more easily between the emerging flux and the surrounding reversed flux than between the two detached poles of the bipoles. And it was likely that the filament erupted more easily between the two poles of bipoles than between the bipoles and the surrounding reversed flux. All the filament eruptions happened during the decay of the emerging flux, which indicated that the activities of magnetic cancellations might be important to the eruptions of filament.

\section{Acknowledgements}

We thank the BBSO team and MDI teams for data support. The work is supported by the NSFC under grant 10573033. 